\title{
UTILIZAÇÃO DE REJEITO MAGNÉTICO PARA MOLDAGEM DE TIJOLOS
}

\author{
Marcos Vinicius Agapito Mendes 1,2 \\ André Silva Ribeiro ${ }^{2}$ \\ Douglas de Assunção Oliveira ${ }^{2}$ \\ Eloisa Angélica Silva Garcia ${ }^{2}$ \\ Marcella Lourenço Bueno Agapito ${ }^{3}$
}

\section{Resumo}

Os resíduos sólidos são uma realidade da sociedade moderna, contribuindo para consequências no planejamento e gestão de indústrias e centros urbanos. Assim, a reutilização destes resíduos para produção de diversos materiais surge como uma alternativa. A mineração é um setor da economia que descarta grandes volumes de material em barragens de rejeito e pilhas de estéril, tornando-se importante a busca por reutilização destes materiais, visando a minimização de impactos ambientais. Este estudo busca analisar a viabilidade técnica de substituição do solo por rejeito magnético gerado no processamento de rocha fosfática na produção de tijolos solo-cimento. Ensaios de resistência mecânica à compressão, absorção e aferição da densidade foram realizados. Os resultados indicam tijolos com resistência mecânica e densidade superior e absorção inferior ao tijolo moldado para controle. Portanto, este estudo preliminar permite concluir que é possível a substituição parcial do solo por rejeito magnético na produção de tijolos solo-cimento, obtendo características semelhantes ou melhores.

Palavras-chave: Rejeito magnético; Resistência mecânica; Tijolo solo-cimento.

\section{USE OF MAGNETIC TAILING FOR BRICK MOLDING}

\begin{abstract}
Solid waste is a reality of modern society, contributing to consequences in the planning and management of industries and urban centers. Thus, the reuse of these wastes for the production of various materials appears as an alternative. Mining is a sector of the economy that discards large volumes of material in tailings dams and sterile piles, making it important to search reuse of these materials in order to minimize environmental impacts. This study search to analyze the technical feasibility of soil substitution by magnetic tailings generated in phosphate rock processing in the production of soil-cement bricks. Tests of mechanical resistance to compression, absorption and density measurement were performed. The results indicate bricks with mechanical resistance and superior density and inferior absorption to the molded brick for control. Therefore, this preliminary study allows to conclude that it is possible the partial replacement of the soil by magnetic waste in the production of soil-cement bricks, obtaining similar or better characteristics.
\end{abstract}

Keywords: Magnetic tailing; Mechanical resistance; Soil-cement brick.

\section{INTRODUÇÃO}

Os resíduos sólidos são uma realidade da sociedade moderna. Trazem sérias consequências para o planejamento e gestão de indústrias e centros urbanos. O patamar de produção sustentável pregado pela sociedade impede que os resíduos sejam descartados de qualquer maneira, sendo necessário sua gestão e planejamento para descarte em locais controlados.
A população mundial cresce em ritmo acelerado nas últimas décadas. Dados publicados no relatório da ONU [I] apontam que a população mundial irá saltar de 7,6 bilhões para 8,6 bilhões até 2030. Outro fator importante é a parcela da população de vive nos grandes centros urbanos, podendo chegar a $66 \%$ em 2050 [2].

'Departamento de Engenharia de Minas, Universidade Federal de Goiás - UFG, Catalão, GO, Brasil.

2Departamento de Mineração, Serviço Nacional de Aprendizagem Industrial - SENAl, Catalão, GO, Brasil. E-mail: marcos.vinicius.agapito@gmail.com ${ }^{3}$ Colégio Estadual Maria das Dores Campos, Catalão, GO, Brasil.

2176-1523 (c) 2019 Associação Brasileira de Metalurgia, Materiais e Mineração. Publicado pela ABM. Este é um artigo de acesso aberto distribuído sob os termos da licença Creative Commons CC BY-NC-ND (Attribution-NonCommercial-NoDerivs) - https:// creativecommons.org/licenses/by-nc-nd/4.0\%. 
Com o crescimento da população, a gestão pública dos centros urbanos se vê pressionada a destinar de forma correta toneladas de resíduos sólidos. O problema se torna caótico com falta de planejamento. A reciclagem é uma opção para minimizar a quantidade de resíduos descartado. Entretanto, o relatório do IPEA [3] deixa claro que este procedimento não é prática comum na sociedade, onde a maioria dos materiais ainda são descartados.

A indústria também é um importante gerador de resíduos sólidos. No Brasil, o progresso socioeconômico vivido nos últimos anos promoveu a inclusão social da população e consequente aumento no consumo de bens materiais. Isto implica o aumento de impactos ambientais de uma produção industrial acelerada e geração de grandes quantidades de resíduos, historicamente descartados de maneira inadequada no país [4].

A Política Nacional de Resíduos Sólidos [5], promulgada em 2010, foi um importante marco regulatório para a preservação do meio ambiente. Visa a gestão integrada e o gerenciamento ambientalmente adequado dos resíduos sólidos. Este instrumento acirrou os deveres das indústrias com a preservação do meio ambiente, tentando evitar o descarte inadequado de resíduos sólidos.

A mineração é um setor da economia que extraí e movimenta grandes volumes de materiais, gerando, inevitavelmente, resíduos sólidos. São descartados de forma controlada em pilhas de estéril e barragens de rejeito, além de outros depósitos de materiais menos comuns. $O$ custo de armazenamento e monitoramento destes resíduos é alto e deve ser realizado ao longo de toda vida útil do empreendimento mineiro.

O processamento de rocha fosfática é classificado pelo IPEA [6] como o terceiro maior gerador de resíduos sólidos na mineração, ficando atrás somente do minério de ferro e ouro. Na rota de processamento para concentração do mineral apatita e produção fertilizantes, aproximadamente $35 \%$ da alimentação de uma usina de beneficiamento torna-se rejeito magnético, $30 \%$ rejeitos de flotação e $12 \%$ lama, ambos descartados em barragens de rejeito [7-10].

A busca por alternativas de reutilização dos resíduos tornou-se uma prática comum diante da sustentabilidade pregada pela sociedade moderna. Estudos com resíduos são comuns, buscando sempre a minimização do volume descartado, dos recursos naturais explorados e preservação do meio ambiente [I I].

No setor de construção civil, o tijolo ecológico tornou-se uma tendência na busca pela sustentabilidade [12]. Diversos materiais são apontados como proposta de estudo na substituição dos componentes principais [13-17]. Fabricados normalmente de solo-cimento, onde os insumos são de fácil obtenção, possuem processo de produção simples e rápido [18]. Não necessitam de processo de queima, como tijolos comuns de argila.

Este estudo tem como objetivo analisar tecnicamente tijolos de solo-cimento fabricados com rejeito magnético gerado no processamento de rocha fosfática, sendo realizados ensaios de resistência mecânica à compressão, absorção e obtenção da densidade.

É pertinente a busca por alternativas de utilização dos resíduos sólidos gerados na mineração, minimizando os impactos ambientais causados pelo descarte de grandes volumes em pilhas de estéril e barragens de rejeito. Além disso, tijolos ecológicos com materiais alternativos permitem redução no custo de produção.

\section{METODOLOGIA}

Para a produção dos tijolos solo-cimento, o primeiro passo consistiu na escolha dos materiais, seguindo o proposto pela norma NBR 10833 [19], com algumas adaptações. Foi utilizado cimento Portland CP II E 32 da marca Cimentos Montes Claros, do grupo Lafarge, além de água potável da torneira. Para seleção do solo, foi realizado um ensaio de distribuição granulométrica de acordo com a NBR NM 248 [20] para verificação das características de granulometria exigidas pela norma NBR 10833 [19]. Também foi realizada uma análise visual do solo, evitando-se a coleta de material com presença de matéria orgânica.

As dimensões dos tijolos seguiram de forma adaptada o proposto pela norma NBR 849I [2 I]. A Figura I apresenta um esquema da fôrma utilizada na moldagem dos tijolos, cujas dimensões são $180 \times 100 \times 38$ milímetros.

O traço utilizado foi de 1:8 (cimento-solo), procedendo-se a moldagem de tijolos de controle, somente com solo, e tijolos com substituição parcial de $20 \%$ do solo por rejeito magnético. $O$ traço dos tijolos com substituição pelo material alternativo foi de $1: 1,6: 6,4$ (cimento-rejeito magnético-solo). Foram moldados 7 tijolos para os ensaios de resistência mecânica à compressão aos sete dias e 3 para ensaios de absorção.

Para a moldagem dos tijolos, todos os materiais utilizados estavam secos. A dosagem dos materiais nas proporções estabelecidas pelo traço foi realizada, procedendo-se a homogeneização com o auxílio de uma enxada. A água foi adicionada a mistura em pequenas proporções, até atingir a umidade ideal (Figura 2).

Após o amassamento dos materiais, a mistura foi adicionada a fôrma. Com o auxílio de uma chapa de aço na parte superior da fôrma e uma prensa hidráulica manual, a mistura foi comprimida dentro da fôrma até atingir a pressão indicada de aproximadamente I tonelada. O tijolo moldado foi então retirado da fôrma com o auxílio de leves golpes no fundo da fôrma com uma marreta de borracha.

Os tijolos removidos da fôrma foram acondicionados em uma chapa metálica (Figura 3), onde permaneceram em processo de cura, livre da ação do calor e intempéries, até atingirem a idade de ruptura. Os tijolos foram umidificados três vezes ao dia por submersão em um tanque com água. Antes da realização dos ensaios de ruptura e absorção, todos os tijolos foram medidos, permitindo posteriormente obter dados de densidade. 
O ensaio de resistência mecânica à compressão foi realizado de forma adaptada ao proposto pela norma NBR 8492 [22]. Os tijolos, após passarem por processo de cura, aos sete dias de sua moldagem, foram posicionados na prensa, entre duas chapas metálicas. Foi inserida pressão a composição até a ruptura dos tijolos. Uma prensa hidráulica

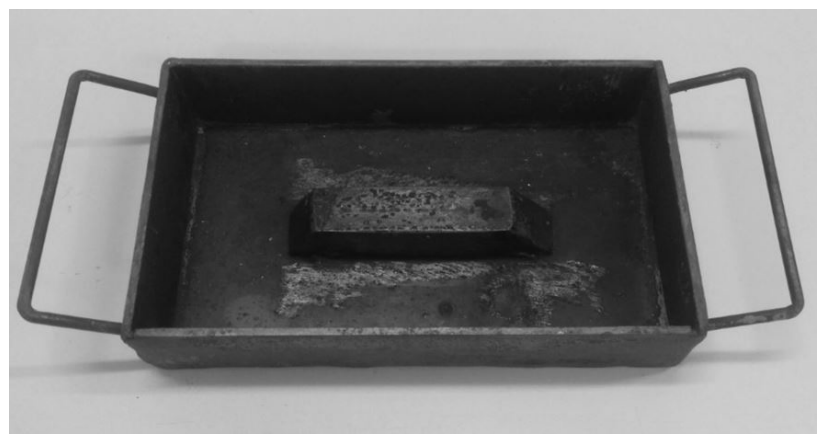

Figura I. Fôrma utilizada na moldagem dos tijolos.

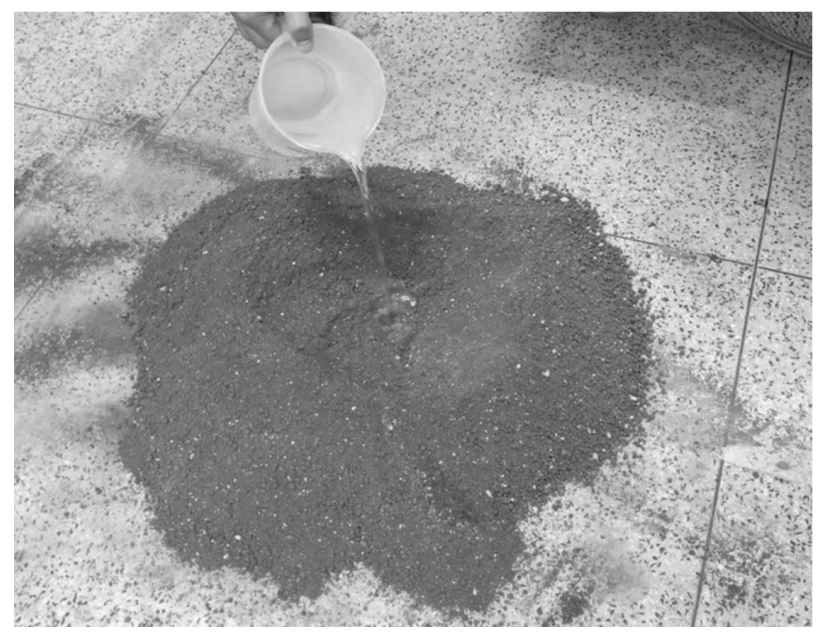

Figura 2. Dosagem dos materiais e adição de água para o amassamento da mistura.

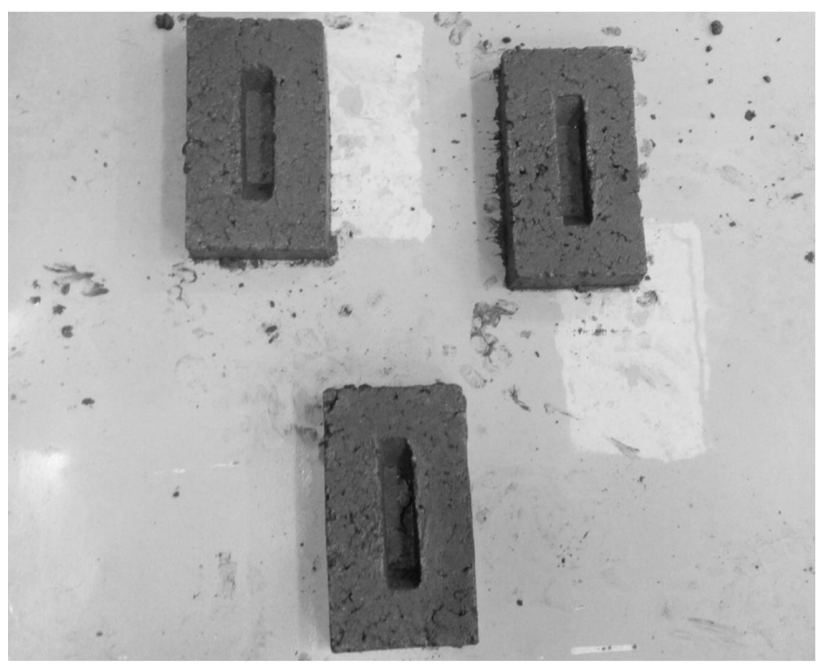

Figura 3. Tijolos acondicionados em chapa metálico após o processo de moldagem. manual com capacidade de 15 toneladas foi utilizada. Por este fato, todos os ensaios de ruptura foram realizados pelo mesmo operador, tentando-se manter uma uniformidade na inserção de pressão aos tijolos, reduzindo erros operacionais.

Os ensaios de absorção dos tijolos seguiram o proposto pela norma NBR 8492 [22]. O primeiro passo consistiu em colocar os tijolos no processo de secagem em estufas, com temperatura controlada entre 105 e $110^{\circ} \mathrm{C}$, onde permaneceram por 24 horas. Após este período, a massa seca foi obtida e os tijolos adicionados em tanques com água, onde permaneceram imersos por mais 24 horas. Posteriormente, foi obtida a massa úmida, permitindo a mensuração da absorção de água dos tijolos moldados.

\section{RESULTADOS}

A análise granulométrica do material é importante para entender o comportamento de tamanho das partículas em diferentes faixas. A Figura 4 apresenta a curva de distribuição granulométrica do solo utilizado, ensaio realizado de acordo com a norma NBR NM 248 [20]. Os dados apresentam 100\% do material passante na peneira de abertura $4,75 \mathrm{~mm}$, e aproximadamente $7 \%$ passa na peneira de abertura $0,075 \mathrm{~mm}$.

Os ensaios de resistência mecânica à compressão simples revelam que os tijolos com substituição parcial de solo por rejeito magnético apresentam resultados superiores ao tijolo de controle (Tabela I). Considerando a média, a resistência do tijolo com substituição ficou $24,4 \%$ acima do tijolo de controle. Além disso, o desvio padrão dos resultados é baixo, o que demonstra confiabilidade na execução dos ensaios.

A absorção por imersão dos tijolos também é um dado importante. É interessante a obtenção de um tijolo com o mínimo de permeabilidade possível, evitando-se problemas de infiltração nas obras em que são utilizados. O tijolo com substituição obteve resultados inferiores ao controle para os ensaios de absorção (Tabela 2), com 19,2\% menos absorção. Com relação a densidade, o tijolo com substituição apresentou resultados $22,5 \%$ superiores ao tijolo controle.

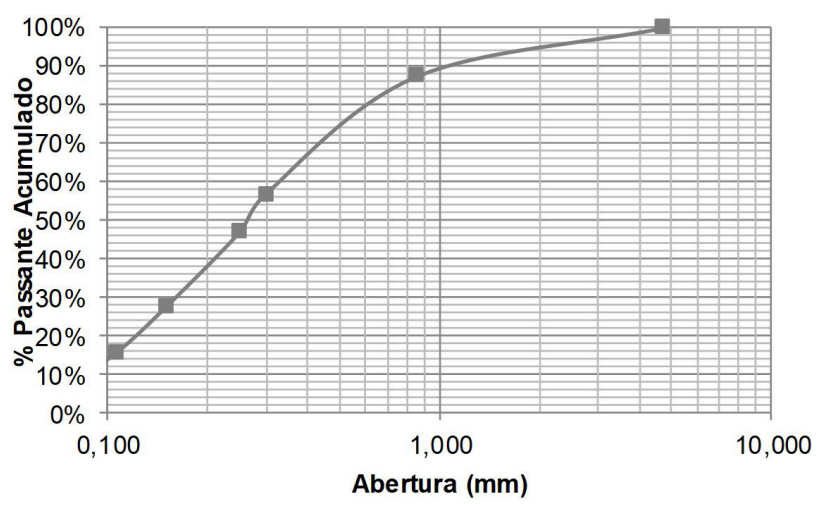

Figura 4. Distribuição granulométrica do solo utilizado na moldagem dos tijolos. 
Tabela I. Resultados de resistência mecânica à compressão simples dos tijolos de controle e com substituição parcial do solo por rejeito magnético

\begin{tabular}{ccc}
\hline \multirow{2}{*}{ Tijolo } & \multicolumn{2}{c}{ Resistência mecânica (Mpa) } \\
\cline { 2 - 3 } & Controle & Substituição \\
\hline 2 & $I, 70$ & $I, 65$ \\
3 & $I, 30$ & $I, 65$ \\
4 & $I, 28$ & $I, 63$ \\
5 & $I, 26$ & $I, 6 I$ \\
6 & $I, 22$ & $I, 6 I$ \\
7 & $I, 10$ & $I, 54$ \\
Média & $I, 00$ & $I, 35$ \\
Desvio & $\mathbf{I , 2 7}$ & $\mathbf{I , 5 8}$ \\
& $\mathbf{0 , 2 0}$ & $\mathbf{0 , 1 0}$ \\
\hline
\end{tabular}

Tabela 2. Resultado dos ensaios de absorção e densidade dos tijolos de controle e com substituição parcial do solo por rejeito magnético

\begin{tabular}{cccccc}
\hline Tijolo & \multicolumn{2}{c}{ Densidade $\left(\mathbf{g} / \mathbf{c m}^{\mathbf{3}}\right)$} & & \multicolumn{2}{c}{ Absorção $(\%)$} \\
\cline { 2 - 3 } \cline { 5 - 6 } & Controle & Substituição & & Controle & Substituição \\
\hline 1 & 1,83 & 2,28 & & 20,12 & 17,30 \\
2 & 1,78 & 2,23 & & 19,49 & 15,54 \\
3 & 1,70 & 2,00 & & 19,28 & 14,73 \\
Média & $\mathbf{1 , 7 7}$ & $\mathbf{2 , 1 7}$ & & $\mathbf{1 9 , 6 3}$ & $\mathbf{1 5 , 8 6}$ \\
Desvio & $\mathbf{0 , 0 5}$ & $\mathbf{0 , 1 2}$ & & $\mathbf{0 , 3 6}$ & $\mathbf{1 , 0 7}$ \\
\hline
\end{tabular}

\section{DISCUSSÃO}

A sociedade vive uma tendência mundial de reutilização de materiais, minimizando impactos ambientais e tornando processos produtivos mais sustentáveis. Os tijolos ecológicos, principalmente aqueles fabricados sem queima (solo-cimento), são alvo de estudos em todo o mundo, com reaproveitamento de variados materiais [23-25].

Neste estudo, os tijolos com substituição parcial de solo por rejeito magnético, foram moldados de forma adaptada. Por este motivo, não é possível comparar a resistência mecânica a compressão com o valor padrão proposto pela norma NBR 849I [2I]. Entretanto, há possibilidade de comparação entre a resistência do tijolo com substituição e de controle, permitindo identificar discrepâncias nos dados.

Visualmente, o rejeito magnético possui granulometria superior ao solo utilizado na moldagem. É possível que as caraterísticas de empacotamento das partículas nos tijolos moldados com substituição foram melhores. Tal fato reflete-se em uma maior massa por volume (densidade superior ao controle), menores taxas de absorção de água (inferior a $20 \%$ ) e maior resistência mecânica.

\section{CONCLUSÃo}

Através dos ensaios de resistência mecânica à compressão, absorção e densidade é possível concluir que há possibilidade de substituição parcial do solo por rejeito magnético na produção de tijolos solo-cimento, com ganhos satisfatórios nas propriedades analisadas. A resistência mecânica foi superior ao tijolo controle e os índices de absorção inferiores e abaixo do padrão estabelecido em norma. Além disso, a densidade, ligeiramente superior ao tijolo de controle, não permite inviabilização em seu uso.

É importante ressaltar que os ensaios foram realizados como análise preliminar. Estudos futuros que busquem variar a porcentagem de substituição, utilizar idades de ruptura maiores e uma melhor análise dos materiais utilizados, é importante.

Mesmo assim, este estudo apresenta um potencial alternativa de reutilização dos rejeitos magnéticos gerados no processamento de rocha fosfática e descartados em barragens de rejeitos, minimizando impactos ambientais. Outro ponto positivo é a possibilidade de utilização destes tijolos em construções comuns devido as boas características apresentadas quando comparado ao tijolo de controle, o que garantiria a redução nos custos.

\section{REFERÊNCIAS}

I United Nations. Department of Economic and Social Affairs, Population Division. World population prospects: the 2017 revision, key findings and advance tables. Working Paper No. ESA/P/WP/248. New York: United Nations; 2018. [acesso em 8 mar. 2018]. Disponível em: https://esa.un.org/unpd/wpp/Publications/Files/WPP2017_KeyFindings.pdf

2 United Nations. Department of Economic and Social Affairs, Population Division. World urbanization prospects: the 2014 revision, highlights (ST/ESA/SER.A/352). New York: United Nations; 2014 [acesso em 8 mar. 20I8]. Disponível em: https://esa.un.org/unpd/wup/Publications/Files/WUP20I4-Highlights.pdf

3 Instituto de Pesquisa Econômica Aplicada - IPEA. Diagnóstico dos resíduos sólidos urbanos: relatório de pesquisa. Brasília: IPEA; 2012. [acesso em 8 mar. 2018]. Disponível em: http://www.ipea.gov.br/portal/images/stories/PDFs/ relatoriopesquisa/ 121009 relatorio_residuos_solidos_urbanos.pdf

4 Instituto de Pesquisa Econômica Aplicada - IPEA. Diagnóstico dos resíduos sólidos industriais: relatório de pesquisa. Brasília: IPEA; 2012 [acesso em 8 mar. 2018]. Disponível em: http://www.ipea.gov.br/portal/images/stories/PDFs/ relatoriopesquisa/I20927_relatorio_residuos_solidos_industriais.pdf 
5 Brasil. Lei $n^{\circ}$ 12.305, de 2 de agosto de 20 I0. Institui a Política Nacional de Resíduos Sólidos; altera a Lei no 9.605 , de 12 de fevereiro de 1998; e dá outras providências. Diário Oficial da União. 2010 [acesso em 8 mar. 20I8]. Disponível em: http://www.planalto.gov.br/ccivil_03/_ato2007-2010/2010/lei/l I2305.htm

6 Instituto de Pesquisa Econômica Aplicada - IPEA. Diagnóstico dos Resíduos sólidos da atividade de mineração de substâncias não energéticas: relatório de pesquisa. Brasília: IPEA; 2012 [acesso em 8 mar. 2018]. Disponível em: http://www.ipea.gov.br/portal/images/stories/PDFs/relatoriopesquisa/I 208 I4_relatorio_atividade_mineracao.pdf

7 Nicoli TA. Proposição de uma nova sistemática de disposição dos rejeitos magnéticos provenientes do beneficiamento da rocha fosfática da mina chapadão, Catalão/GO [dissertação]. Ouro Preto: Universidade Federal de Ouro Preto; 2014.

8 Mendes MVA, Silva AC, Barbosa DHBM. Substituição do agregado miúdo por magnetita na composição do concreto. Enciclopédia Biosfera. 2014;10(19):513-523.

9 Silva AC, Silva EMS, Mendes MVA, Barbosa DHBM. Magnetite tailing replacing quartz sand in concrete formulation. In: Proceedings of $14^{\text {th }}$ International Mineral Processing Symposium; 2014; Kusadasi, Turkey. Istanbul: Turkish Mining Development Foundation; 2014. p. 599-604.

10 Silva AC, Mendes MVA, Silva EMS. Substituição de areia por magnetita na formulação do concreto. Tecnologica em Metalurgia, Materiais e Mineração. 2016;13(3):265-269.

I I Deus RM, Battistelle RAG, Silva GHR. Resíduos sólidos no Brasil: contexto, lacunas e tendências. Engenharia Sanitaria e Ambiental. 20I5;20(4):685-698. http://dx.doi.org/I0.1590/SI4I3-4I5220I5020040I 29347.

12 Weber E, Campos RFF, Borga T. Análise da eficiência do tijolo ecológico solo-cimento na construção civil. Ignis. 2017 [acesso em 8 mar. 2018];6(2):18-34. Disponível em: http://www.periodicosuniarp.com.br/ignis/article/ view/II 78/706

13 Marins DS, Santos JHT, Nascimento BLS, Lima IBG, Santos NM. Resistência a compressão simples e absorção de água em tijolos ecológicos com a incorporação de papel kraft. In: Anais do $7^{\circ}$ Fórum Internacional de Resíduos Sólidos; 2016; Porto Alegre, Brasil. Brasília: Universidade de Brasília; 2016 [acesso em 8 mar. 2018]. Disponível em: http://www.firs.institutoventuri.org.br/images/T097_RESIST\%C3\%8ANCIA_A_COMPRESS\%C3\%83O_ SIMPLES_E_ABSOR\%C3\%87\%C3\%830_DE_\%C3\%8IGUA_EM_TIJOLOS_ECOLL\%C3\%93GICOS_COM_A_ INCORPORA\%C3\%87\%C3\%830_DE_PAPEL_KRAFT.pdf

14 Silva D, Aguiar MB. A utilização da casca da banana como substituição de parte do cimento na produção de tijolos ecológicos: a busca por alternativas sustentáveis. Percurso Acadêmico. 2017 [acesso em 8 mar. 2018];7(I3): 19-32. Disponível em: http://periodicos.pucminas.br/index.php/percursoacademico/article/view/P.2236$0603.2017 \mathrm{v} 7 \mathrm{n} / 3 \mathrm{p} / 9 / 12372$

15 Copari VP, Oliveira MB. Confecção de tijolos ecológicos como alternativa para reutilização dos resíduos gerados por centrais dosadoras de concreto. REINPEC. 2016;2(I): I22-127. http://dx.doi.org/I0.2095 I/2446-6778/v2n la l0.

16 Sena RJ, Laursen A, Silva JS. Avaliação mecânica de tijolo maciço solo-cimento contendo resíduo de pet. Veredas. 2017 [acesso em 8 mar. 2018]; 10(I):69-83. Disponível em: http://veredas.favip.edu.br/ojs/index.php/veredas I/ article/view/643/pdf

17 Santos AM, Passos MG, Santos JHT, Costa FN. Utilização da fibra de ráfia como adição em solo-cimento para produção de tijolos ecológicos: uma avaliação físico-mecânica. Revista Univap. 20I6;22(40):702-707. Edição Especial. https://doi.org/10.18066/revistaunivap.v22i40.1457.

18 Oliveira JL, Silva JC, Damasceno FA, Damasceno LFB. Desenvolvimento de protótipos de prensas manuais para confecção de tijolos ecológicos, visando a sustentabilidade de pequenas construções rurais. Revista Brasileira de Agropecuária Sustentável. 2015 [acesso em 8 mar. 2018];5(2):38-46. Disponível em: http://www.rbas.com.br/index. php/rbas/article/view/295/276

19 Associação Brasileira de Normas Técnicas - ABNT. NBR 10883: Fabricação de tijolo e bloco de solo-cimento com utilização de prensa manual ou hidráulica - Procedimento. Rio de Janeiro: ABNT; 2013.

20 Associação Brasileira de Normas Técnicas - ABNT. NBR NM 248: Agregados - Determinação da composição granulométrica. Rio de Janeiro: ABNT; 2003.

2I Associação Brasileira de Normas Técnicas - ABNT. NBR 849I: Tijolo solo-cimento - Requisitos. Rio de Janeiro: ABNT; 2013.

22 Associação Brasileira de Normas Técnicas - ABNT. NBR 8492: Tijolo solo-cimento - Análise dimensional, determinação da resistência à compressão e da absorção de água - Método de ensaio. Rio de Janeiro: ABNT; 2013. 
Mendes et al.

23 Zhu M, Wang H, Liu L, Ji R, Wang X. Preparation and characterization of permeable bricks from gangue and tailings. Construction \& Building Materials. 20 I7;(I48):484-49I. http://dx.doi.org/I0. I0I6/j.conbuildmat.20I7.05.096.

24 Murmu AL, Patel A. Towards sustainable bricks production: an overview. Construction \& Building Materials. 2018;(165):1 I2-125. http://dx.doi.org/10.1016/j.conbuildmat.2018.01.038.

25 Rodrigues LP, Holanda JNF. Recycling of water treatment plant waste for production of soil-cement bricks. Procedia Materials Science, 20I5(8):197-202. https://doi.org/10.1016/j.mspro.2015.04.064.

Recebido em: 19 Mar. 2018

Aceito em: 20 Set. 2019 